\title{
Stiffness control in under-actuated robotic origamis with shape memory polymer
}

\author{
Amir Firouzeh, Student Member, IEEE, Marco Salerno, Member, IEEE, and Jamie Paik, Member, IEEE
}

\begin{abstract}
Under-actuated systems offer compact designs with easy actuation and control but at the cost of limited stable configurations and reduced dexterity compared to the directly driven and fully actuated systems. Here, we propose a compact origami-based design to control the stable configurations and the overall stiffness of an under-actuated robotic finger by modulating the material stiffness of the joint. The design of the robotic finger is based on the robotic origami design principle in which multiple functional layers are integrated to make a nominally $2 \mathrm{D}$ robot with a desired functionality. To control the stiffness of the structure, we controlled the elastic modulus of a shape memory polymer (SMP) via embedded customized stretchable heater. We monitor the configuration of the finger using the feedback from the customized curvature sensors embedded in each joint. We studied the stable configurations and the contact forces of a finger with 3 joints at different stiffness settings. A scaled down version of the design was used in a gripper with two fingers and different grasp modes were demonstrated through activating different set of joints.
\end{abstract}

Index Terms-Under-actuated robotic finger, robotic origami, adjustable stiffness joints, shape memory polymer, glass transition.

\section{INTRODUCTION}

As the robots move from the confined work space in factories to the unstructured environment of humans, they need to manipulate objects with different shapes and sizes. This requires highly dexterous robots with many degrees of freedom (DoF) [1]. Independent actuation of each DoF in such a system results in a highly dexterous but rather complex robot. The need for lighter and easier to control robots has lead to an alternate approach of using under-actuated mechanisms for activating many DoFs using a single source of actuation [2], [3]. Moreover, the inherent tolerance of the under-actuated hands to impacts and their ability to conform to their environment through distribution of the input actuation between the joints [4] makes them soft and inherently safe for human interaction. Under-actuated robotic grippers can preform different grasping motions depending on the object shape and the contact points [5], [6]. Desired distribution of contact force between the phalanges and the object is achievable through proper assignment of the transmission ratio between the input and each joint [7], [8]. However, once the design of an under-actuated system is set, it will have fixed transmission ratio for the joints and fixed motions. However, different tasks and working environments require different motions and forces at the contact points. Different methods were suggested for switching between modes of operation in under-actuated hands for meeting the requirements of different

A.Firouzeh, M. Salerno, and J. Paik are with the Reconfigurable Robotics Lab, EPFL, Lausanne, Switzerland e-mail: (see http://rrl.epfl.ch/). tasks [4], [9], [10]. Switching between discrete modes of operation rather than fine control over the transmission ratio and requiring an additional source of actuation are two of the main limitations in the proposed methods.

Changing the joint stiffness is an another method for altering the power transmission ratio between the input and different joints. Different methods for directly embedding the compliance in the robot's hardware have been proposed [11][14]. Many of these rely on conventional means of actuation and mechanisms [15]-[17]. In this paper, we use the material properties for changing the stiffness of the joints. Compared to the designs that are based on motors and mechanisms, using material properties results in a more compact and scalable design. The glass transition in thermoplastics [18][22], Jamming [23]-[25], and phase change of wax [26] and metals [27], [28] are among the different methods [29] that use material properties for controlling the stiffness of the structure.

In this research, we use Shape Memory Polymer, SMP (MM5520, SMP Technologies), layer for controlling the stiffness of Robogami joints. As a thermoplastic, SMP displays considerable change in its mechanical properties around the glass transition temperature [30]. It also has the added advantage of high shape recovery. These properties along with easy processing and fabrication make SMP a viable choice for variable stiffness bodies and joints of robots. For the structure of the finger, we present a compact design based on the layer by layer fabrication process of robotic origamis, Robogamis. Different functional layers are integrated to construct the Robogami finger with desired functions such as sensing ,actuation, and stiffness modulation. We use a scaled down design of the robotic finger in a gripper with adjustable grasp modes to demonstrate the design versatility and the scalability of the Robogami design and fabrication technique.

The main contributions of this work are:

- Understanding the relationship between the stiffness of the SMP joints and the stable configurations in a tendon driven under-actuated Robogami. This will allow activating and controlling of different modes of operation.

- Introducing the joint stiffness control method based on material properties. Combined with the Robogami layerby- layer manufacturing methodology, the proposed stiffness control scheme results in a scalable and adaptable design framework for under-actuated and high DoF robots.

- Studying the configurations of an under-actuated finger with three joints at various input control parameters (tendon displacement and the temperature of the SMP layers) through the joint angle feedback. The customized sensing solutions for temperature and joint angle are 


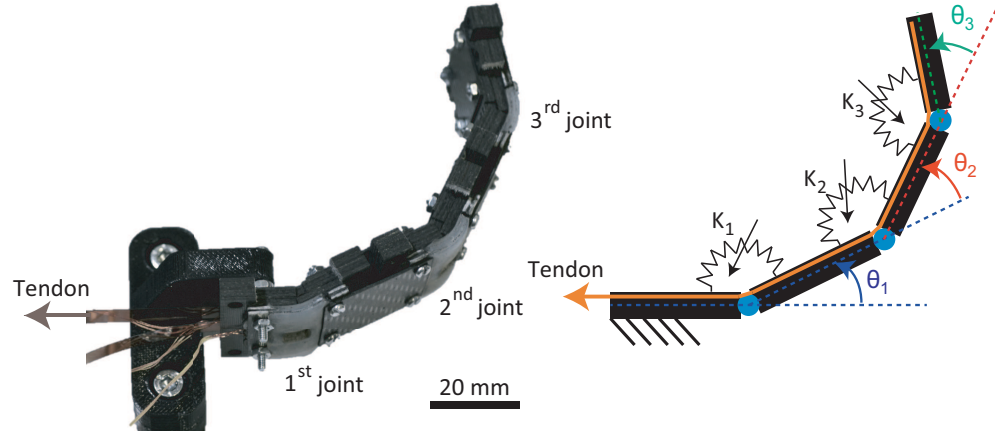

(a) (b)

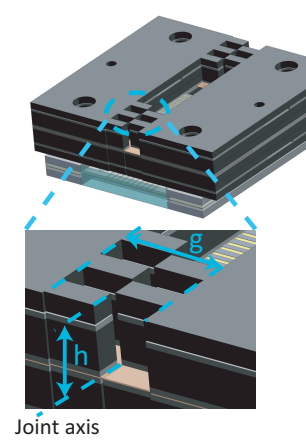

(c)

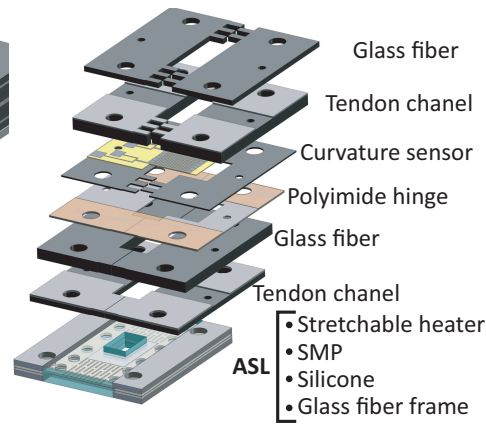

(d)

Fig. 1: The Robogami finger with adjustable stiffness joints and the construction of a single joint. (a) The finger consists of three joints with adjustable stiffness and a tendon that drives the joints. (b) Schematic of the finger depicting the joint positions and their adjustable stiffness. (c) Each joint is designed as a stand alone module. The castellated pattern in the module places the axis of rotation on the polyimide sheet and at a constant distance from the ASL. (d) Different functional layers are integrated using the layer by layer manufacturing process to make each module.

compatible with the origami structure and demanding operating conditions of Robogamis.

In the next Section, we present the design of the Robogami finger and study the relation between the joints' stiffness and finger configuration. In Section III, we present the design of the ASL and the change of the heater electrical resistance and ASL stiffness with temperature. In Section IV, the design of the curvature sensors and their characterization result are presented. In Section V, we find the remaining parameters in the model and compare the configuration of a finger with three joints with the predicted and estimated configurations from the model and the curvature sensors respectively. We also study the overall stiffness of the finger and its effect on the contact forces in the enveloping motion around an object. Finally in Section VI, we evaluate exemplary different grasp modes in a gripper that uses a scaled down version of the proposed joints to confirm the feasibility of the proposed design for activating different synergies in under-actuated systems.

\section{ROBOGAMI JOINT DESIGN AND KINETOSTATIC STUDY OF THE UNDER-ACTUATED FINGER}

Robogamis are constructed by integrating multiple functional layers to build quasi-2D structures. The design of the robotic finger based on this fabrication process allows us to embed different layers in a thin structure: hinge, tendons, curvature sensors, and ASLs. Fig. 1a presents the overview of the Robogami finger consisting of three joints and Fig. $1 \mathrm{~b}$ illustrates the schematic of the finger depicting the tendon route and the adjustable stiffness joints. In this design, we fabricated individual modules that are assembled together using bolts and nuts to have the option of using and interchanging different number of joints. Fig. 1c presents the design of a single module. Different functional layers are stacked together to make each module as presented in Fig. 1d. The cured glass fiber layers are used as the structural material in this design. Each of these layers is processed using a UV laser micro machining station (detailed process and machine specifications are presented in [31]). In the hinge area, we used a castellated pattern to fix the axis of rotation and to increase the lateral stability of the joint. The hinge axis in this design falls between the tips of the castellated structure from the two tiles and on the polyimide hinge layer. The gap between the tips of the castellated design should be small to keep the axis of rotation fixed and on the polyimide layer and at a constant distance from the ASL. Still this distance should be large enough to ensure the mechanical endurance of the polyimide hinge layer in repeated cycles. In the present design, this distance was set to $50 \mu \mathrm{m}$ which yielded robust and repeatable motion. By modulating the length, $g / 2$, and the height, $h$, of the teeth (Fig. 1c) we can set joint limits. We designed the joints to have $90^{\circ}$ limit on one side based on (1).

$$
\theta_{\text {JointLimit }}=\sin ^{-1}\left(\frac{g}{2 h}\right)
$$

Here we study one directional motion of the finger. So on the ASL side, we designed the glass fiber layers without the castellated pattern. The distance between the tiles on this side is the same as the laser beam spot size of $50 \mu \mathrm{m}$. This puts the joint limit on this side at less than $1^{\circ}$. So when the tendon is released the elastic force applied by the ASLs returns the joints to their limit and the finger to the straight configuration.

The correlation between the tendon displacement and the bending angle in each joint governs the transmission ratio between the input force to the tendon and the torque applied to each joint. According to Fig. 2, (2) gives the contribution of each joint to the displacement of the tendon:

$$
X_{i}=g-\sqrt{{X_{i_{x}}{ }^{2}+X_{i_{y}}{ }^{2}}^{2}}
$$

In (2), $X_{i}$ is the contribution of the $i^{\text {th }}$ joint to the tendon displacement and $X_{i_{x}}$ and $X_{i_{y}}$ are its components as depicted in Fig. 2 which are calculated as:

$$
\begin{aligned}
& X_{i_{x}}=g / 2\left(1+\cos \theta_{i}\right)-b \sin \theta_{i} \\
& X_{i_{y}}=g / 2\left(\sin \theta_{i}\right)+b \cos \theta_{i}-b
\end{aligned}
$$

The geometrical parameters in (3), (4), and the following equations are depicted in Fig. 2 and their values are presented in Table I. The relation between the joint angular speed and the tendon speed is calculated using (2)-(4) as: 


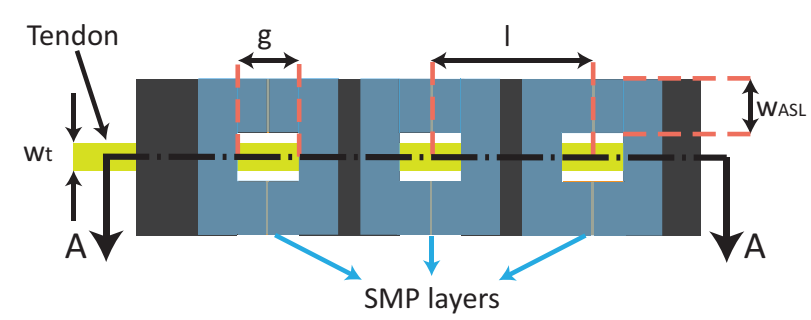

(a)

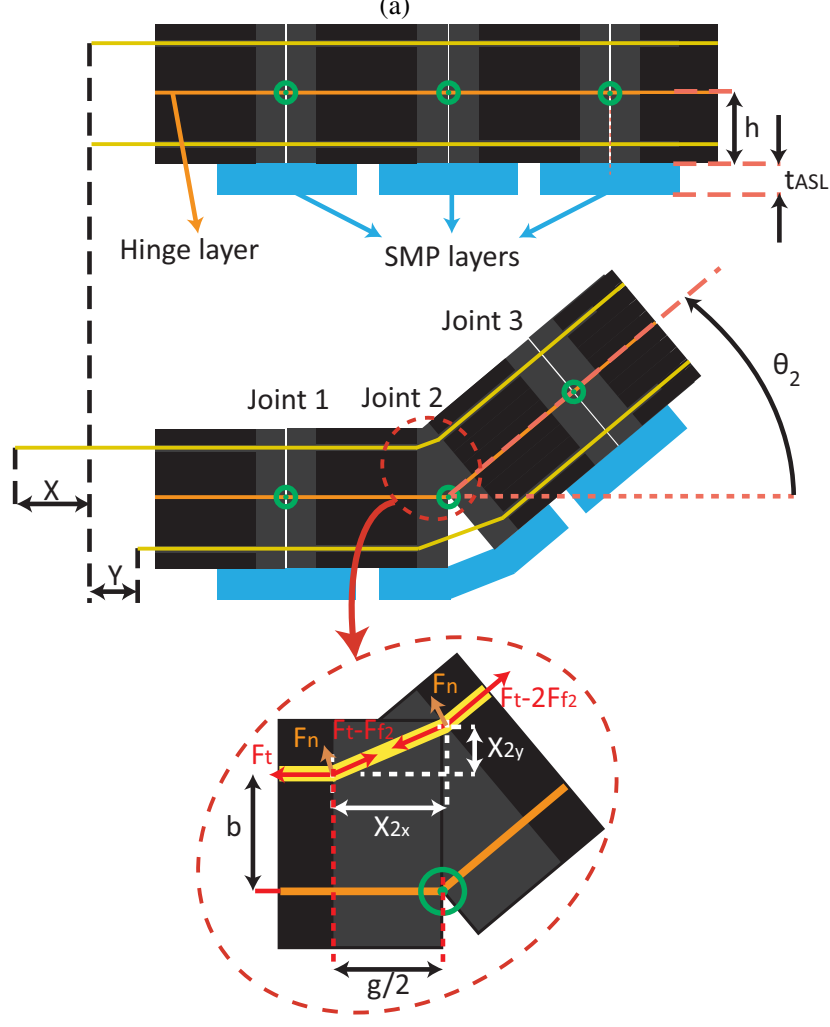

(b)

Fig. 2: Schematic of the finger presenting the design parameters and the actuation of the second joint. (a) The top view. (b) The side view of the cross section A-A depicted in Fig. 2a. The schematic of the finger with its second joint at an angle $\theta$ is presented to highlight the displacement of the tendon and the length change of the ASL. The point of interaction between the tendon and the glass fiber layer is magnified to show the contact forces that result in frictional losses.

$$
d X_{i}=\frac{X_{i_{x}} b+X_{i_{y}} \frac{g}{2}}{\sqrt{{X_{i_{x}}{ }^{2}+X_{i_{y}}^{2}}^{2}}} d \theta_{i}
$$

We should point out that the displacement, $X$, from (2) corresponds to the effective displacement that results in the configuration change. The input displacement, however, is the sum of this effective displacement and the tendon elongation due to the tensile load. So for the total input displacement, $X_{\text {total }}$, we have:

$$
X_{\text {total }}=\sum_{i=1}^{n} X_{i}+\sum_{i=1}^{n} \frac{F_{t}-\sum_{j=1}^{i} 2 F_{f_{j}}}{k_{i}}
$$

The first term in (6) accounts for the effective displacement and the second term for the elongation of the tendon. $k_{i}$ in this equation is the stiffness of each section of the tendon between
TABLE I: The values of the design parameters. The thickness of different layers in this table can have upto $10 \%$ discrepancy caused by the difficulties in controlling the thickness of different layers in the composite.

\begin{tabular}{|c|c|c|}
\hline Parameters & Value $(\mathrm{mm})$ & Description \\
\hline$l$ & 34 & Phalanx length \\
\hline$g$ & 4 & Gap size in the middle part \\
\hline$w_{A S L}$ & 7 & ASL width in the active part \\
\hline$l_{A S L}$ & 4 & ASL length in the active part \\
\hline$w_{t}$ & 6 & Tendon width \\
\hline$t_{A S L}$ & 2 & ASL thickness \\
\hline$h$ & 2.4 & ASL distance from the axis of rotation \\
\hline$b$ & 2 & Tendon distance from the axis of rotation \\
\hline
\end{tabular}

the two joints. Here we considered the decrease in the tendon force, $F_{t}$, from the base to the tip due to the friction forces at each joint, $F_{f_{j}}$, and calculated the elongation for each segment between the joints separately. There are two contact points per joint as presented in Fig. 2b. We considered the friction force for both contact points to be equal. This force is dependent on the tendon force and the joint angle and is calculated as:

$$
F_{f_{i}}=\mu_{f} F_{n_{i}}+C_{f_{i}} F_{t}=\left(\mu_{f} 2 \sin \left(\theta_{i} / 4\right)+C_{f_{i}}\right) F_{t}
$$

In (7), $\mu_{f}$ is the friction coefficient and $F_{n_{i}}$ is the normal contact force between the tendon and its channel. The second term term in this equation accounts for the friction forces due to the misalignment in the tendon channel.

To study the stable configurations of the finger at different stiffness settings for the joints, the kinetostatic analysis of under-actuated fingers presented by Brigle and Gosselin [32] is adopted and modified to account for the energy storage in the ASL and the friction losses. Equating the input work and the work done by the finger on the environment and the stored energies in the hinge and the ASLs, we have:

$$
\boldsymbol{F}_{\boldsymbol{t}}^{T} \dot{\boldsymbol{X}}=\sum_{i=1}^{n} \boldsymbol{\xi}_{\boldsymbol{i}} \circ \boldsymbol{\zeta}_{\boldsymbol{i}}+W_{A S L}
$$

In (8), the left side is the input work minus the friction losses. The elements of $\boldsymbol{F}_{\boldsymbol{t}}$ are the tension in the tendon at each joint and the elements of $\dot{\boldsymbol{X}}$ are the rate of the tendon displacement caused by the motion of each joint. The first term in the right side of this equation is the reciprocal product of the screws corresponding to the twist, $\xi_{i}$, and the wrench, $\zeta_{i}$, at the contact point on each phalanx. The second and third terms on the right side, $W_{A S L}$ correspond to the work done to deform the ASLs. For the contact point with the object, we neglected the friction forces and only considered the normal forces. So the work done by the contact forces is calculated as:

$$
\sum_{i=1}^{n} \boldsymbol{\xi}_{\boldsymbol{i}} \circ \boldsymbol{\zeta}_{\boldsymbol{i}}=\boldsymbol{f}^{T} \boldsymbol{J} \dot{\boldsymbol{\theta}}=\boldsymbol{f}^{T}\left[\begin{array}{cccc}
d_{11} & 0 & \ldots & 0 \\
d_{12} & d_{22} & \ldots & 0 \\
: & : & : & : \\
d_{1 n} & d_{2 n} & \ldots & d_{n n}
\end{array}\right] \dot{\boldsymbol{\theta}}
$$

In (9), $\mathbf{f}$ is the vector of the contact forces, $d_{i j}$ is the distance of the $i^{\text {th }}$ joint from the contact force vector applied to the $j^{\text {th }}$ phalanx, and $\dot{\boldsymbol{\theta}}$ is the vector of the joints' rotational speed. 
The tendon force at each joint, elements of $\boldsymbol{F}_{\boldsymbol{t}}$ in (8), is calculated by subtracting from the input tension all the friction forces from the base to that joint.

$$
\begin{aligned}
& \boldsymbol{F}_{\boldsymbol{t}}^{T}=
\end{aligned}
$$

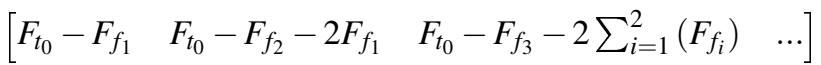

The rate of the tendon displacement, $\dot{\mathbf{X}}$ in (8), has the following relation with the angular velocities of the joints:

$$
\dot{\mathbf{X}}=\boldsymbol{T} \dot{\boldsymbol{\theta}}=\left[\begin{array}{cccc}
\frac{\partial X_{1}}{\partial \theta_{1}} & 0 & \ldots & 0 \\
0 & \frac{\partial X_{2}}{\partial \theta_{2}} & \ldots & 0 \\
: & : & : & \vdots \\
0 & 0 & \ldots & \frac{\partial X_{n}}{\partial \theta_{n}}
\end{array}\right] \dot{\boldsymbol{\theta}}
$$

$\frac{\partial X_{i}}{\partial \theta_{i}}$ in this equation is the transmission ratio between the input and the $i^{t h}$ joint. This is calculated from (5) which corresponds to the relation between the tendon displacement rate and the angular velocity of a desired joint when all the other joints are locked in position.

The stored energy in the ASL, $W_{A S L}$, is found as:

$$
W_{A S L}=\boldsymbol{F}_{\boldsymbol{A S L}}{ }^{T} \dot{\boldsymbol{\Delta}}=\left(\boldsymbol{K}_{\boldsymbol{A S L}} \boldsymbol{\Delta}\right)^{T} \dot{\boldsymbol{\Delta}}
$$

In (12), $\boldsymbol{\Delta}$ and $\boldsymbol{F}_{\boldsymbol{A} S \boldsymbol{L}}$ represent the elongation and the force of the ASLs. $\boldsymbol{K}_{\boldsymbol{A} S \boldsymbol{L}}$ in this equation is the matrix of the joint stiffness which has the following form:

$$
\boldsymbol{K}_{\boldsymbol{A S L}}=\left[\begin{array}{cccc}
C_{k_{1}} F_{k_{A S L}}\left(T_{1}\right) & 0 & \ldots & 0 \\
0 & C_{k_{2}} F_{k_{A S L}}\left(T_{2}\right) & \ldots & 0 \\
: & : & : & : \\
0 & 0 & \ldots & C_{k_{n}} F_{k_{A S L}}\left(T_{n}\right)
\end{array}\right]
$$

In (13), $F_{k_{A S L}}(T)$ is the ASL stiffness as a function of temperature. This function is determined using the tensile test results for the ASLs. $F_{k_{A S L}}(T)$ provides the trend of the stiffness change with temperature. Due to the variation between different ASL samples and Robogami modules (mainly the variation in the thickness of the layers) we expect some variation in the stiffness of the joints which is accounted for using the correction factors $\left(C_{k_{i}}\right)$ in (13).

The ASL elongation, $\Delta$, is a function of the joint angle and is calculated as:

$$
\boldsymbol{\Delta}=2\left(h+\frac{t_{A S L}}{2}\right) \sin \frac{\boldsymbol{\theta}}{2}
$$

Replacing the stored energy in the ALSs and the hinges in (8) yields the following set of equations that correlate the tension in the tendon with the joint angles and their stiffness:

$$
\boldsymbol{F}_{\boldsymbol{t}}^{T} \boldsymbol{T}=\boldsymbol{f}^{T} \boldsymbol{J}+\left(\left(h+\frac{t_{A S L}}{2}\right)^{2}\left(\boldsymbol{K}_{\boldsymbol{A S L}} \sin \boldsymbol{\theta}\right)^{T}\right)
$$

(15) determines the configuration of the robotic finger for an assigned set of joints' stiffness and the tension in the tendon. This set of equations along with (6) is used to evaluate the required tendon displacement. In the next Section we examine the relation between the stiffness of the ASL and its temperature and in Section $\mathrm{V}$ we characterize the remaining parameters in the model and compare the model prediction with the test results.

\section{THE ASL CONSTRUCTION AND ITS STIFFNESS VARIATION WITH TEMPERATURE}

The modulus of elasticity of thermoplastic polymers change orders of magnitude around their glass transition point. This makes thermoplastics a good choice for adjustable stiffness layers for controlling the elastic properties of the Robogami joint. In this research, we use a shape memory polymer that has the advantage of higher strain recovery over normal thermoplastics. To regulate its temperature, we embedded a $70 \mu \mathrm{m}$ thick stretchable heater in the SMP layer. The fabrication process of the heater and its integration with SMP are presented in [18]. The overview of this process is presented in Fig. $3 a$ and $3 b$.

The residual strain in the SMP layer highly affects the stiffness of the joints and hence the repeatability of the motion. To achieve a higher shape recovery, we embedded the SMP layer inside silicone rubber. To do so, we first embedded the SMP layer between two glass fiber layers as presented in Fig. $3 \mathrm{c}$. The glass fiber layers act both as the frame for attaching the ASLs to the joints and also as the mold for casting the silicone layer. Fig. 3d presents the final form of the ASL. The holes in the SMP layer presented in Fig. $3 b$ will shape silicone columns that would transfer the force between the silicone and the SMP (the design details are in [22]).

In order to regulate the stiffness of the joints, we need to control the temperature of the SMP layer. we use the electrical resistance of the heaters which provides a measure

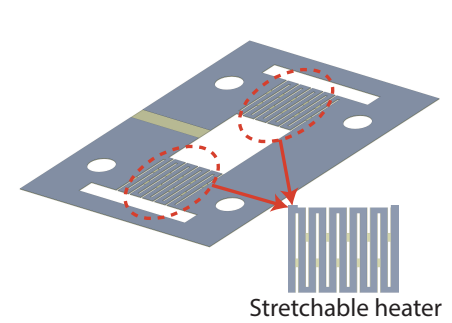

(a)

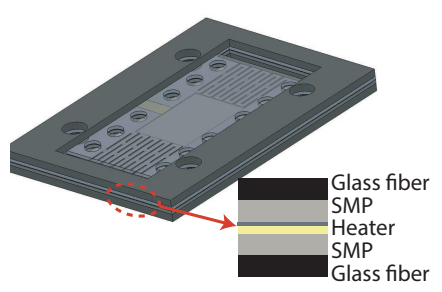

(c)

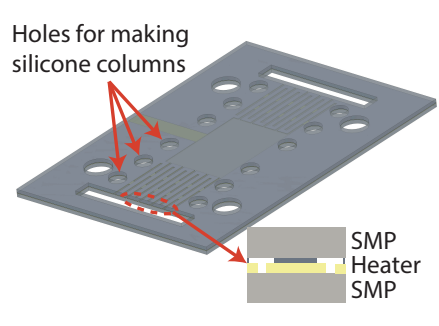

(b)

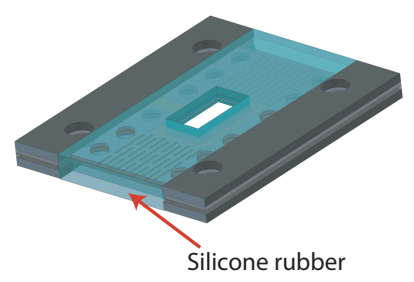

(d)
Fig. 3: The overview of the fabrication process of the ASL. (a) The schematic of the stretchable heater layer. (b) The heater is embedded in SMP. (c) The SMP layer in the glassfiber frame. (d) The final module with silicone rubber enveloping the SMP layer. 


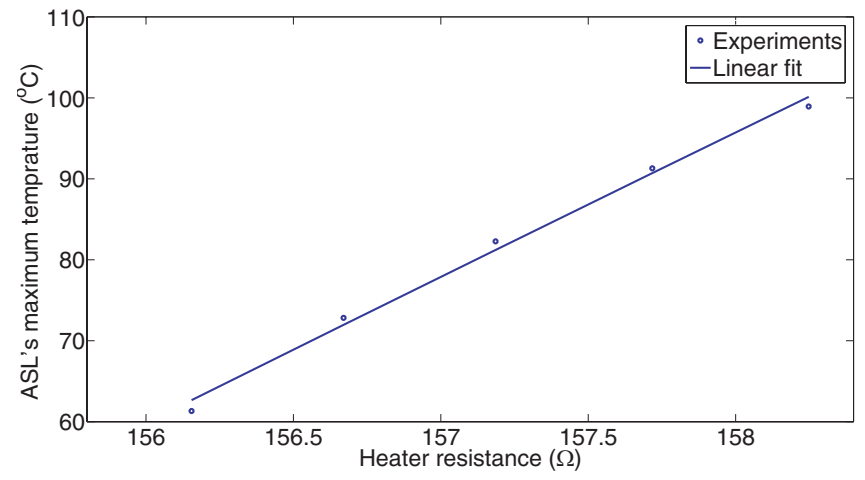

Fig. 4: The ASL temperature vs. the heater electrical resistance. The electrical resistance has a linear correlation with the temperature.

of the average temperature of the SMP layer as the thermal sensor. Using the heaters as temperature sensors simplifies the fabrication process by reducing the number of components.

We studied the relation between the electrical resistance of the heater and its temperature at different set-points using a thermal camera. The test results, presented in Fig. 4, shows a linear relation between the resistance of the heater and the temperature of the ASL, with $R^{2}$ of 0.99 . We should point out that for each heater we have slightly different sensitivity due to small fabrication differences. We calibrate the temperature sensitivity of each heater using the thermal camera reading by setting the desired temperature to the maximum set point $\left(110^{\circ} \mathrm{C}\right)$ and varying the sensitivity factor till reaching similar estimation for the temperature from the thermal camera and the heater. There is a considerable temperature gradient in the ASL and we used the maximum temperature reading from the thermal camera in characterization and calibration of the heaters.

To characterize the stiffness of the ASL as a function of its temperature, we used the feedback from the heater and assigned different temperatures to the ASL $\left(30{ }^{\circ} \mathrm{C}\right.$ to $110^{\circ} \mathrm{C}$ with $10{ }^{\circ} \mathrm{C}$ increments). At each temperature, the sample was loaded three times upto $2.5 \mathrm{~mm}$ or $15 \mathrm{~N}$, whichever occurs first. We approximated the elastic behavior of the ASL with a linear function at each temperature and reported the slope as the stiffness of the sample. Fig. 5 presents the average stiffness and the standard deviation at each measurement point (12 tests per point). The result of the characterization tests is used as a lookup table in the model to evaluate the stiffness of the ASL as a function of its temperature $\left(F_{k_{A S L}}(T)\right.$ in (13)).

The SMP that we used in the ASLs has the glass transition temperature at $55^{\circ} \mathrm{C}$ (MM5520, SMP Technologies). In calibrating the temperature sensors, we used the temperature of the surface of the silicone which is lower than the temperature of the SMP layer. This is the reason for the abrupt stiffness change between $40{ }^{\circ} \mathrm{C}$ and $50{ }^{\circ} \mathrm{C}$ in Fig. 5 instead of the expected sharp drop between $50{ }^{\circ} \mathrm{C}$ and $60{ }^{\circ} \mathrm{C}$.

Due to the variation in the sample thickness, there is a discrepancy between the silicone surface temperature, used in calibration, and the effective temperature of the SMP layer for each sample. Moreover, we observed that the resistance of the same sample can have a small drift over time which can lead to

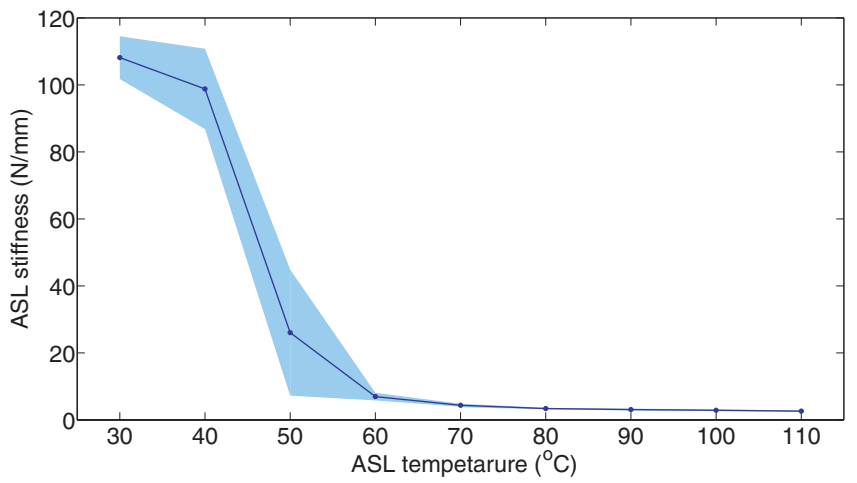

Fig. 5: The stiffness of the ASL layer as a function of its temperature. The shaded area shows the standard deviation of the data points.

errors in the temperature set point even for the same sample in different cycles of loading. The inaccuracies in controlling the temperature along with the high temperature sensitivity of the stiffness around the glass transition temperature leads to a large standard deviation around the transition temperature. This problem can be alleviated in future by using polymers with more gradual modulus of elasticity change with temperature.

According to Fig. 5, the stiffness of the ASL changes more than 40 times in the entire temperature range. The stiffness drops more than 15 times between $30{ }^{\circ} \mathrm{C}$ and 60 ${ }^{\circ} \mathrm{C}$. Although the rate of the stiffness change with temperature is considerably lower at temperatures higher than $60{ }^{\circ} \mathrm{C}$, this region is still useful for controlling the stiffness and the stable configuration of the under-actuated finger.

In this Section, we presented the design of the ASLs and demonstrated temperature control using the electrical resistance of the heaters. We confirmed more than 40 times change in the stiffness of the ASL which will be used to control the joint stiffness in the under-actuated Robogami.

\section{LOW PROFILE CURVATURE SENSORS FOR MONITORING JOINT ANGLE IN ROBOGAMI STRUCTURES}

Given the variation in the stiffness of different samples reported in the previous section, we need the feedback from the joint angles to monitor the trajectory and to adjust the control parameters accordingly. The compact design of the Robogami structures and the geometrical constrains necessitate the design of a custom-made curvature sensor for this application. The customized curvature sensor functions based on the resistance change of a metal path under strain. To induce unidirectional strain in the metal layer under bending deformation, we used a laminate of metal and polyimide. To increase the resistance change of the sensor, a serpentine path was etched in the metal layer. The schematic of the sensor is presented in Fig. 6a. Constantan was chosen as the metal layer based on the low sensitivity of its electrical resistance to temperature change. The sensor is fixed at one end and its other end slides in and out of the adjacent tile while following its bending angle (Fig. $6 b)$. Allowing this sliding motion reduces the maximum strain in the metal layer and prevents plastic deformation and failure of the sensors. 


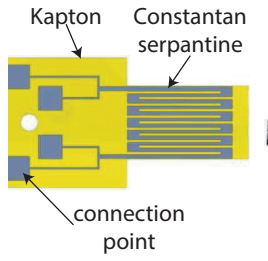

(a)

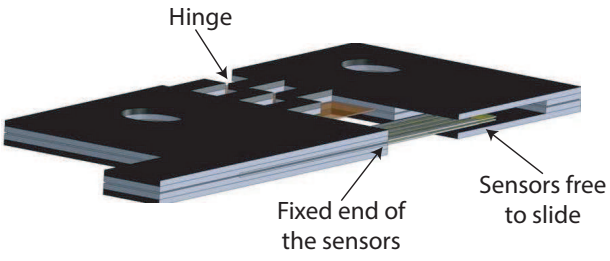

(b)

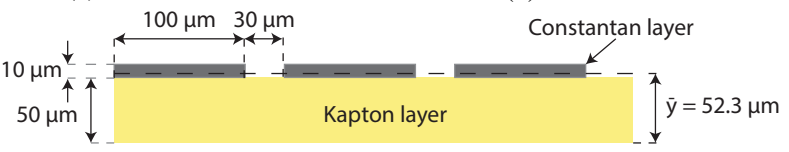

(c)

Fig. 6: The low profile curvature sensor for Robogami structures. (a) The schematic of the sensor which comprises a Constantan serpentine $(10 \mu \mathrm{m}$ thick) laminated on $50 \mu \mathrm{m}$ thick Polyimide. When the sensor is bent, the metal layer mainly undergoes tension or compression depending on the bending direction. This loading condition results in increase or decrease in the overall resistance of the sensor. (b) The overview of the assembly of the two sensors in the Robogami joint. The sensors are fixed on one tile and are free to slide in and out of the second tile. (c) Schematic of the curvature sensor cross section.

To reduce the maximum strain for a given bending angle, and maximizing the elastic range of deformation, thin metal and Polyimide layers were used in the laminate, $10 \mu \mathrm{m}$ and $50 \mu \mathrm{m}$ respectively. For this laminate, the neutral plane falls $2.3 \mu \mathrm{m}$ above the metal and Polyimide interface which puts the metal layer (as presented in Fig. 6c) partly in tension and partly in compression under bending loads. Ideally using a thinner metal layer for having the entire Constantan volume in either tension or compression is preferable (we used $10 \mu \mathrm{m}$ Constantan layer based on the availability). Considering the gauge factor of 2.0 for Constantan, the resistance change can be evaluated by the following equation:

$$
\delta R=2 R \bar{\varepsilon}
$$

In (16), $R$ is the overall resistance of the sensor, and $\bar{\varepsilon}$ is the average strain in the metal layer. Considering a simple bending, the strain in the metal layer is calculated as:

$$
\varepsilon=\frac{(y-\bar{y})}{\rho}, \rho=\frac{l_{\text {sensor }}}{\theta}
$$

In (17), $y-\bar{y}$ is the distance from the neutral plane and $\rho$ is the radius of curvature. $l_{\text {sensor }}$ is the length of the sensor and $\theta$ is the bending angle. Given the dimensions that are presented in Fig. 6c, the average strain in the metal layer is calculated as:

$$
\bar{\varepsilon}=3.8 \times 10^{-4} \theta
$$

According to (16) and (18), the expected sensitivity, $\delta R / R$, is $7.6 \times 10^{-4} / \mathrm{rad}$. Because of the low sensitivity, we need to use precise electronics and 4-point measurement for avoiding inaccuracies related to contact and wiring resistances.

The stiffness of the joint is controlled through temperature adjustment. Given the design of the joint and the close proximity of the curvature sensors and the heating elements, as presented in Fig. 1, we expect a significant temperature change in the sensors. Although Constantan has a low sensitivity to

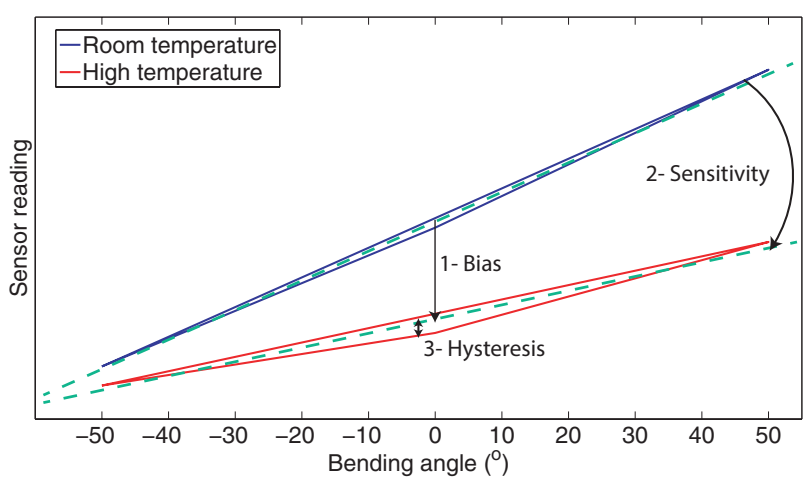

Fig. 7: The 3 major effects of temperature on the sensor reading. 1) The negative bias in the reading (caused by the negative electrical resistancetemperature coefficient of Constantan). 2) The decrease in the sensitivity of the sensor. 3) The increase in the hysteresis loop width. This figure is an schematic representation of the sensor reading and the effects are not to the scale.

temperature variation, given the wide temperature range, the thermal effects are not negligible. To account for these effects, we placed two sensors with the same pattern back to back which puts the metal layers in opposite loading conditions. Given the thin profile of the two sensors, we expect them to have similar thermal condition. Subtracting the resistance of the two sensors is expected to cancel out the effect of the temperature on resistance. We define the joint angle indicator, $I_{\theta}$, as:

$$
I_{\theta}=R_{1}-R_{2}
$$

Ideally we expected the joint angle indicator to be independent of the temperature. The test results, however, suggests that the temperature change affects the resistance in three different ways: 1- adding a bias to the sensor reading which is canceled in the joint angle indicator, 2- changing the sensitivity, and 3increasing the hysteresis in the sensor reading. These three effects are presented in the exaggerated schematic of Fig. 7. The overall resistance of each sensor can be written as:

$$
R_{\text {sensor }}=b(\text { Temp })+K_{\theta}(\text { Temp }) \theta \pm h y s(\text { Temp })
$$

In (20), $b($ Temp $)$ is the bias in the sensor reading, $K_{\theta}$ is the sensitivity of the sensor which as discussed is a function of the temperature, and hys(Temp) represents the hysteresis. Subtracting the resistance of the two sensors cancels out the bias in the temperature reading but it does not compensate for the sensitivity change and the hysteresis. So the joint angle indicator has the following form:

$$
I_{\theta}=R_{1}-R_{2}=2 k_{\Theta}(\text { Temp }) \theta \pm 2 h y s(\text { Temp })
$$

The sensitivity change which is a function of the temperature can be accounted for based on the temperature of the sensors. We estimate the temperature of the two sensors using a temperature indicator, $I_{T e m p}$. This is calculated by adding the resistances of the two sensors for canceling out the strain effect. 


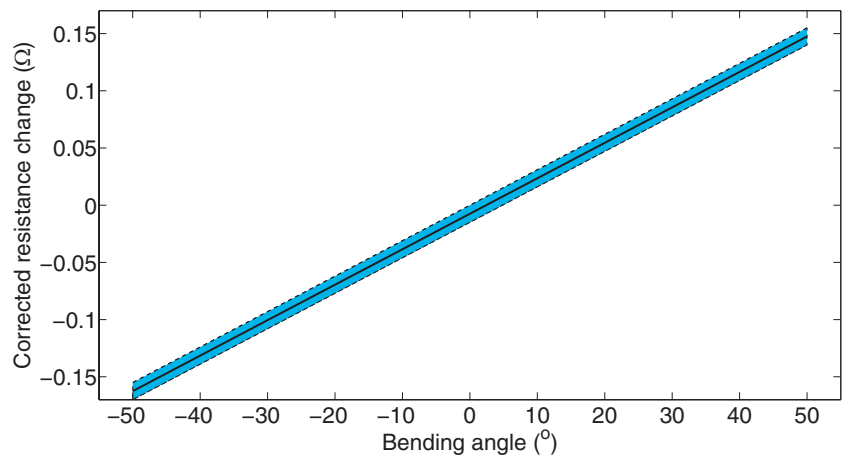

Fig. 8: The corrected response of the two sensors versus the bending angle. The bias caused by the temperature is canceled out by subtracting the resistance of the two sensors. The reading was also corrected to account for the change in the sensitivity due to the temperature change. The sensor is tested in 25 loading cycles with different amplitudes. The results are fairly repeatable and linear and an envelope of $\pm 2.4^{\circ}$ contains the data from all the loading cycles.

$$
I_{T e m p}=\frac{R_{1}+R_{2}}{2}
$$

We approximated the change of the sensitivity, $K_{\Theta}$, with a linear function of the temperature. To characterize the rate of sensitivity change, we tested a module in its maximum range of motion while the heater in the SMP layer was activated and the temperature of the curvature sensors was increasing. The result of this test was used to determine a linear correction for $K_{\Theta}$ as a function of $I_{T e m p}$ which was used in all subsequent tests. The last term in (21) can not be canceled easily. However, the temperature indicator can provide a measure of how prominent the hysteresis effect is. In the characterization tests, the hysteresis in the sensor reading was negligible.

To study the response of the curvature sensor, it was tested till different maximum amplitudes $\left(10,20,30,40\right.$, and $\left.50^{\circ}\right)$ with $10^{\circ}$ steps. The tests were repeated 5 times for each maximum amplitude. The sensor was characterized in a complete joint with the SMP layers. The thermal effects are canceled out using the reading from the two complementary sensors to make the response compatible with the sensor reading at the room temperature. Fig. 8 presents the characterization test results. The sensor response is fairly linear. The sensor sensitivity is $8.5 \times 10^{-4} / \mathrm{rad}$ which is higher than the estimated value. The difference can be attributed to variation in the thickness of the metal layer and its gauge factor. The sensor response is fairly repeatable and an envelope of $\pm 2.4^{\circ}$ contains the data from all the tests. The result of this section confirmed accurate joint angle estimation using the proposed sensors. In the next section, we use these sensors to estimate the configuration of the finger and validate this estimation using the result of processing the video of the motion.

\section{UNDER-ACTUATED ROMOGAMI FINGER}

Through stiffness modulation of the joints in under-actuated systems, we can control the stable configurations of the robot and its interaction forces with the environment. In this Section, we study the free displacement of an under-actuated finger consisting of three mod ules at different stiffness settings for the joints. We demonstrate the possibility of controlling the configuration of the finger, hence the position and the orientation of its end effector. This enables us to apply forces at the desired position and orientation for manipulating objects. We also study the under-actuated grasping motion of the finger during which it conforms to an object. We show that by controlling the stiffness of the joints we can control the magnitude of the contact force that the finger produces before it pulls out and looses contact. This enables us to switch between soft mode for working in sensitive environments and stiff mode for applying larger forces when necessary.

In the first part of this Section, we characterize the remaining parameters in the model: the stiffness correction factors, the friction forces, and the stiffness of the tendon. In the second part, we use the model of the finger to predict its configuration at different temperature settings and compare the model prediction with the actual configuration and the curvature sensor estimation. In the third part, we study the contact forces in grasping a fixed object at different stiffness settings for the finger.

\section{A. Characterization and calibration of the model parameters for the Robogami finger}

The model presented in Section II predicts the configuration of the finger based on the temperature setting for the ASLs. Here, We determine the parameters in the model that are affected by the assembly of the finger by individually actuating the three joints of the finger. These parameters are: the tendon stiffness, the friction forces, and the correction factor for the joint stiffness, $C_{k_{i}}$.

There are two sources of friction losses in the Robogami finger: 1- the friction between the castellated features of the adjacent tiles, 2- the friction between the tendon and its channel. The first source of friction is independent of the force in the tendon and based on the tests on a sample without the ASL, it is negligible. The second source of the friction is significant and is accounted for in the model as presented in (7). The magnitude of this force depends on the joint angle and the tendon tension. To study this force and to find the friction coefficient for each joint, we activated one ASL in the finger at $110{ }^{\circ} \mathrm{C}$ and left the other two ASLs at the room temperature. After reaching the thermal equilibrium, we applied tendon displacements in the range of $0-4 \mathrm{~mm}$ with 0.5 $\mathrm{mm}$ increments. As presented in Fig. 9, when the direction of the motion reverses, there is a sudden drop in the tendon tension. This drop is caused by the friction force changing direction. Due to the elasticity of the tendon, this drop is not completely vertical. The slope of this change depends on the tendon length and it varies for different joints. The drop in tensile force $\left(\Delta F_{t}\right)$ is due to the accumulated friction forces from the base to the active joint and is calculated as:

$$
\Delta F_{t}=2\left[F_{t}\left(\mu_{f} 2 \sin \left(\theta_{i} / 4\right)+\sum_{j=1}^{i} C_{f_{j}}\right)\right]
$$




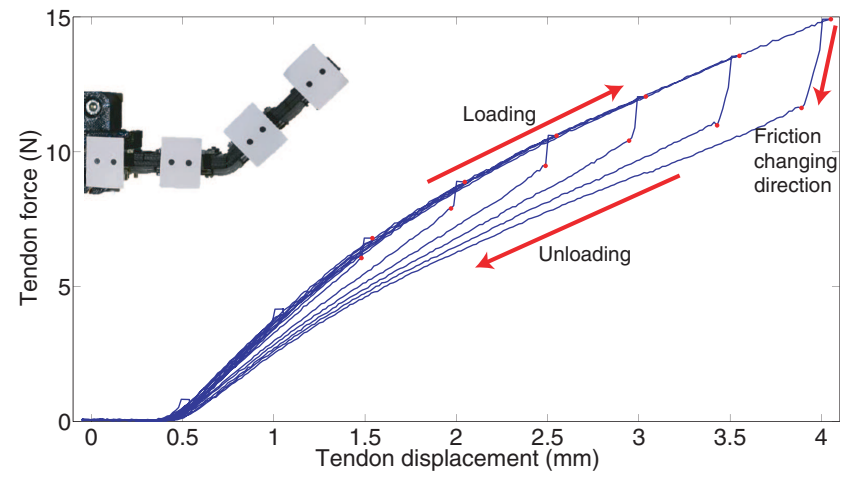

Fig. 9: Tension in the tendon as a function of the tendon displacement. We test each joint in the finger individually at the maximum temperature in order to characterize the friction force. The sudden drop in the tendon tension when the direction of the motion is reversed corresponds to the change in the direction of the friction force between the tendon and the glass fiber channel. The corresponding bending angle for each point of displacement is found from the video of the tests. A snapshot of the video corresponding to the characterization test for the second joint is presented in this figure. The markers on each phalanx is to facilitate the video processing.

The friction force changes direction when the direction of the motion reverses. So the drop in the tendon tension is twice of the friction force which is accounted for by the factor of two in (23). Based on the characterization results the parameters of the friction force were found as 0.23 for $\mu_{f}$ and $0.01,0.011$, and 0.014 for $C_{f_{1}}, C_{f_{2}}$, and $C_{f_{3}}$ respectively. To find these parameters, the joint angles corresponding to each displacement were determined from the video processing.

The second set of parameters to be determined are the correction factors for the joint stiffness. In the model, we use the stiffness-temperature correlation presented in Fig. 5 $\left(F_{k_{A S L}}(T)\right)$ to determine the stiffness of the joints at different temperatures. The correction factor for the stiffness of the ASL $\left(C_{k_{i}}\right)$ which is introduced in (13) accounts for the differences between the ASLs and the height of the modules. To find the correction factors, each joint of the finger was tested individually at different temperature settings (from $30{ }^{\circ} \mathrm{C}$ to $110{ }^{\circ} \mathrm{C}$ with $10{ }^{\circ} \mathrm{C}$ increments). At each temperature, the tendon was pulled till reaching 15 N. Fig. 10 presents the

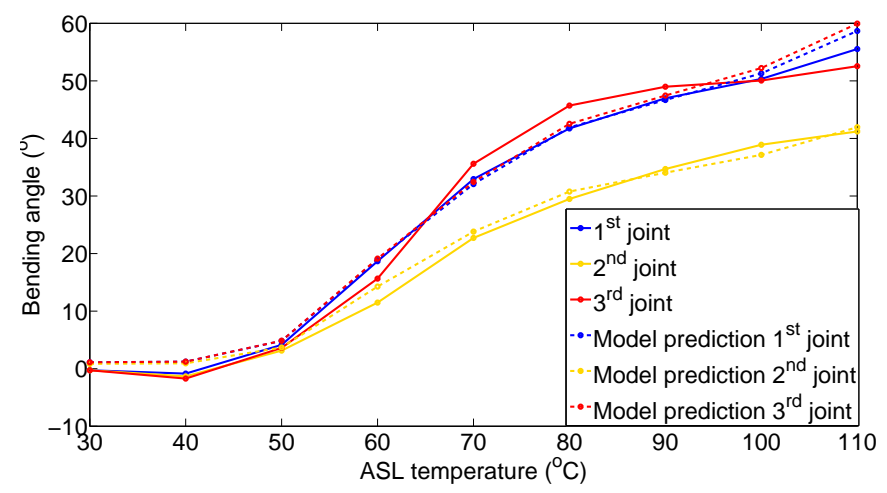

Fig. 10: The bending angle for the three modules at different temperature settings (each module was tested separately). The model was corrected based on the experimental results by applying a correction factor to the ASL stiffness $\left(C_{k_{i}}\right)$ that accounts for variation in the thickness of different layers. maximum bending angle at each temperature setting. The first and the third joints in the finger have similar behavior but the second joint bends less for the same tendon force which indicates that the second joint is stiffer than the other two joints. Based on the test results we found the following correction factors: 1.14, 1.48, and 1.12 for the first, the second, and the third joints, respectively.

The elongation of the tendon under tensile load is not negligible and is accounted for in the model (6). To evaluate the stiffness of the tendon, we used the force displacement relation in the test with all ASLs at $30{ }^{\circ} \mathrm{C}$. The results confirmed linear elastic behavior for the tendon with the stiffness coefficient of $10.7 \mathrm{~N} / \mathrm{mm}$ for the full length of the tendon, $142 \mathrm{~mm}$. We use this to calculate the stiffness of the segments of the tendon between each two joints which is used in (6).

In this Section, we determined the tendon stiffness, its friction with the channel, and the stiffness correction factor for the ASLs. The model that was introduced in Section II along with these parameters are used to predict the behavior of the under-actuated finger in the following Sections.

\section{B. Robogami finger configuration control through adjusting joint stiffness}

The stable configuration of the finger can be controlled by assigning proper stiffness to the joints. In this Section, we compare the model prediction and the sensor estimation with the actual configuration of the finger at different temperature settings for the ASLs. In all of the tests in this section, the tendon was pulled till reaching $4.5 \mathrm{~mm}$ displacement or $15 \mathrm{~N}$ tensile force (whichever happened first). We started the tests with the ASL in all joints at $110{ }^{\circ} \mathrm{C}$. The configuration of the finger at this temperature setting is presented in Fig. 11a. The phalanx angle which is presented in this figure is the sum of the joint angles from the base to each phalanx. The friction force between the tendon and its channel decreases the tension in the tendon from the base to the tip. This results in larger moment for the joints closer to the base. So with the same stiffness we expect larger bending angles for the joints closer to the base which is concordant with the results of Fig. 11a. Next we decreased the temperature of the base joint to $40{ }^{\circ} \mathrm{C}$ in $10{ }^{\circ} \mathrm{C}$ increments. At each step, the finger was actuated twice. Reducing the temperature of the first joint results in smaller bending angle for this joint and larger angle for the other two joints (with the same tendon displacement). The second case in Fig. 11a shows an intermediate step with the first joint at $70{ }^{\circ} \mathrm{C}$ and the other two at $110{ }^{\circ} \mathrm{C}$. Due to the friction forces, the bending angle of the second joint in this case is larger than the third joint in spite of its higher stiffness (Fig. 10). After the temperature of the first joint was decreased to $40{ }^{\circ} \mathrm{C}$, the tests were continued by decreasing the temperature of the middle joint to $40{ }^{\circ} \mathrm{C}$ in $10{ }^{\circ} \mathrm{C}$ increments. As expected, the second joint angle decreases as it becomes stiffer in the lower temperatures. The third case in Fig. 11a shows an example with the first joint at $40{ }^{\circ} \mathrm{C}$ and the other two joints at $100{ }^{\circ} \mathrm{C}$ and $110{ }^{\circ} \mathrm{C}$. Fig. $11 \mathrm{a}$ also presents the comparison between the sensor estimation (dashed line) and 


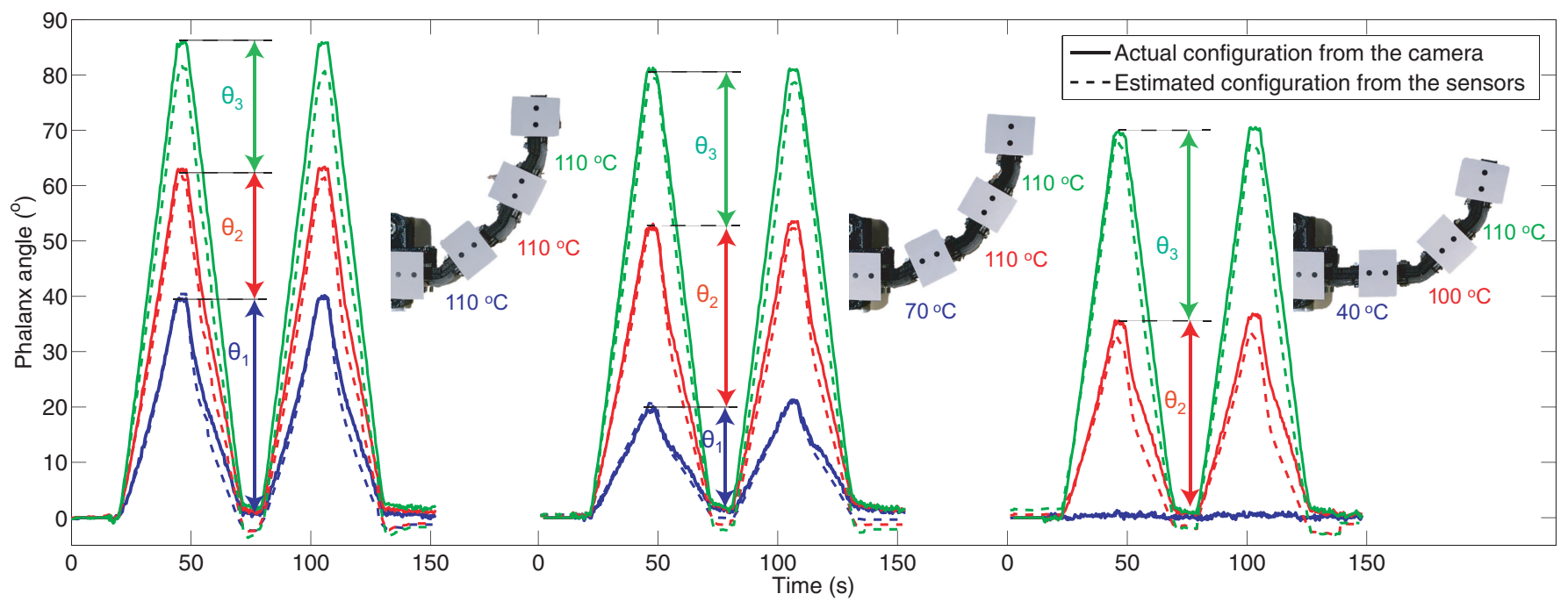

(a)

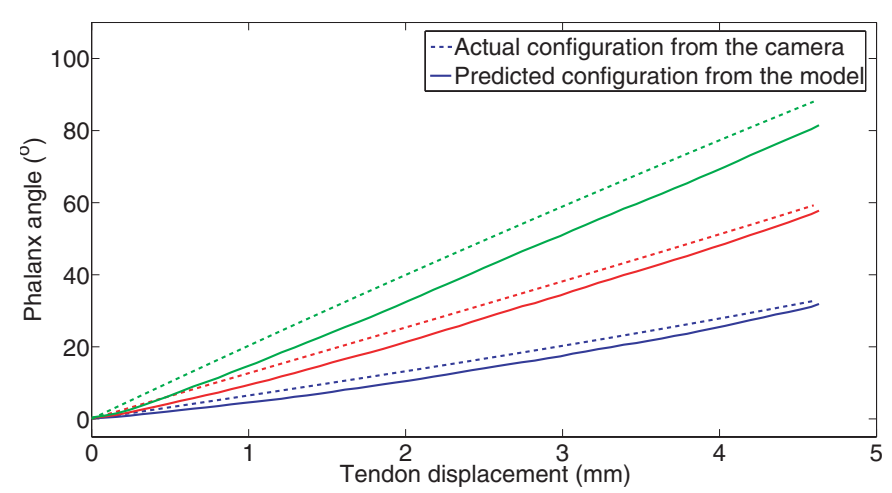

(b)

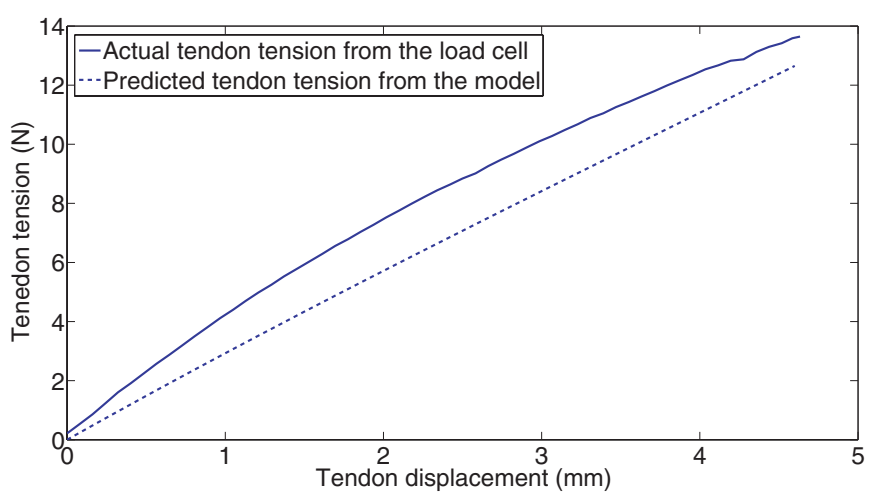

(c)

Fig. 11: The free motion of the Robogami finger at different temperature settings. (a) The angle of the 3 phalanges of the finger from their initial position at different temperature settings. The dashed line is the curvature sensor reading and the solid line is the output of the video processing. The joint angle (the difference between the angles of two adjacent phalanges) is marked in the plot. The configuration of the finger for each temperature setting with the temperature corresponding to each joint is also presented in this figure. (b) Model prediction for each of the three phalanges and their actual position. The temperature set points in this case are $80{ }^{\circ} \mathrm{C}$ for the $1^{\text {st }}$ joint and $110{ }^{\circ} \mathrm{C}$ for the $2^{\text {nd }}$ and $3^{r d}$ joints. (c) Tendon tension predicted by the model and the actual tension. The temperature set points are the same as part (b).

the actual phalanx angle (solid line). The curvature sensors are able to estimate the configuration of the finger with a high accuracy. The RMS error between the estimated and the actual joint angle at the maximum deformation for the three joints in the 15 tests described here ( 2 repetition for each test) was $1.8^{\circ}$ which guarantees reliable feedback from the embedded sensors.

We also compared the model prediction with the actual configuration of the finger in these tests. The model is able to predict the trend in the configuration change at different temperatures. Fig. $11 \mathrm{~b}$ presents the comparison between the model prediction and actual configuration. The RMS error between the model prediction and the actual joint angle for all the tests is $4.6^{\circ}$. Fig. 11c compares the tension in the tendon with the predicted value from the model which shows around $7 \%$ error at the maximum load. The trend for the force displacement relation predicted by the model is rather linear since we have adopted a linear model for the elastic behavior of the ASL. However, The actual force displacement relation is nonlinear and to have a better prediction in future we need to use a more accurate model for the elastic behavior of the ASL.

The test results confirmed the feasibility of configuration control through modulating the temperature of the SMP layers. The model is able to predict the configuration of the finger at different temperature settings and can be used to assign the temperature of the ASLs in the joints for reaching a desired configuration. The errors that are caused by model inaccuracies can be corrected by adjusting the control command for the tendon displacement and ASL temperature set points using the curvature sensors feedback.

\section{The overall stiffness control of the Robogami finger}

We can control the overall stiffness of the finger by modulating the stiffness of its joints. Using this feature, we can operate it in its soft mode in the sensitive environments with limited applied contact forces and in its stiff mode for handling heavy loads or performing precision grasp. Here, we study the motion of the under-actuated finger with different joint stiffness as it conforms to the shape of a fixed object and apply contact 


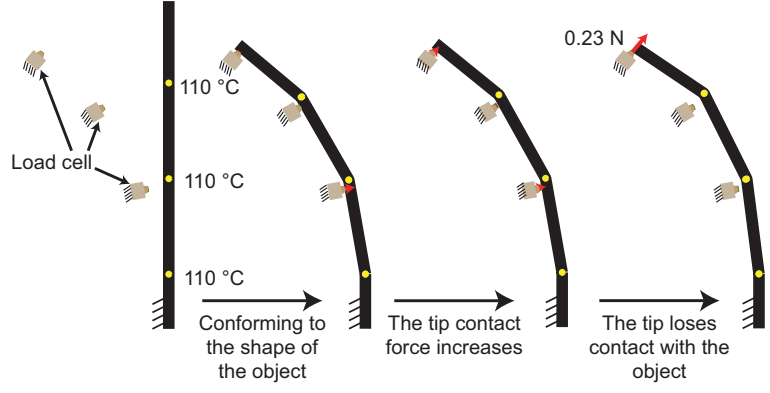

(a)

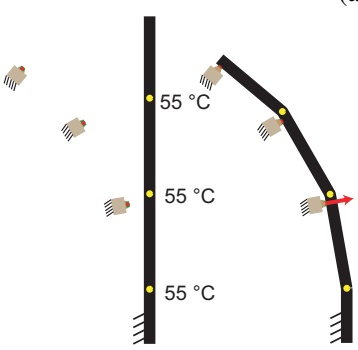

(b)
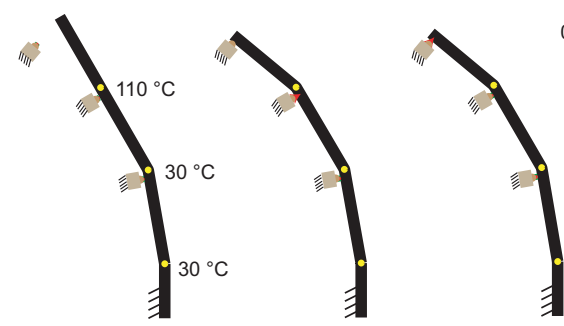

(c)

Fig. 12: The simulation result for the contact forces between the finger and the object at different joint stiffness settings. (a) In this case the temperature of all joints are set to the maximum, $110^{\circ} \mathrm{C}$, and the finger is in its softest state. By pulling the tendon, the finger conforms to the shape of the object and starts applying forces. Increasing the tendon displacement increases the contact force at the tip and finally causes the finger to deform at the first and second joints which results in loosing contact at the tip. (b) In this case the temperature of all joints are set to $55{ }^{\circ} \mathrm{C}$. The motion of the finger in this case is similar to the previous case but the contact forces are larger. (c) In the third case only the third joint is activated. The other two joint angles are set to put the last phalanx at a desired position and orientation. The maximum contact force in this case is larger since the first and second joints are stiffer compared to the other two cases.

forces. We have considered three different cases. In the first case, all three joints are activated at $110{ }^{\circ} \mathrm{C}$ and the finger is in its softest state. Next all the joints are activated at $55^{\circ} \mathrm{C}$. We expect the ratio of the stiffness of the joints and hence the motion of the finger to be similar in these two cases with only difference being the contact force magnitudes. Finally we study the case where the first two joints are fixed in position and only the tip joint is moving. In this mode of operation, the initial deformation puts the tip phalanx at the desired position and orientation. By activating only one joint at this state, we expect larger forces compared to the other two cases.

Fig. 12 presents the simulation results for the finger in these three cases. We consider one contact point per phalanx. We have placed the contact point at $2 \mathrm{~mm}$ distance from the tip when the finger initially conforms to the object and study the forces that it can produce before the deformation in the first and second joints would cause the third phalanx to loose contact with the load cell. For the first and the second case, as tendon is pulled, the finger conforms to the shape of the object, start applying forces to the load cells, and finally looses its contact with the object. The configuration of the finger for the first two cases are very similar while the maximum contact force at the tip just before loosing contact changes from $0.23 \mathrm{~N}$ to $0.7 \mathrm{~N}$ by increasing the stiffness of the finger as presented in Fig. 12a and Fig. 12b. For the third case the finger is initially actuated in its soft mode to conform to the shape of the object and then the first and second joints are locked in this position. The third joint is the only active joint in this case and as demonstrated by the simulation results, Fig. $12 \mathrm{c}$, the maximum force before the finger looses its contact is increased to $0.95 \mathrm{~N}$.

To verify the simulation results a 3D printed stand with the one-directional force sensor (FSS015WNGX, Honeywell) with the same orientation was fabricated and the same scenarios of the simulation were replicated. The maximum force for the three cases was measured to be: $0.17,0.32$, and $0.75 \mathrm{~N}$. While the measured forces confirms the trend observed in the simulation, their values are smaller in comparison. This difference is caused by the combination of positioning errors for the object and the deformation in the load cells' 3D printed stand.

Controlling the overall stiffness and the maximum forces that an under-actuated system can produce is a desired feature in robot human interaction. Joints with adjustable stiffness can be used in robots for controlling their level of backdrivability and the maximum contact force magnitude for safe interactions in sensitive environments.

\section{UNDER-ACTUATED GRIPPER WITH ADJUSTABLE STIFFNESS JOINTS}

To further study the feasibility of using the Robogami joints with adjustable stiffness for distributing the actuation in underactuated systems, a scaled down version of the joints was designed and used in a gripper with two fingers (Fig. 13a). Each finger has 5 joints with adjustable stiffness. Based on the task, the deformation of each joint and the overall stiffness of the fingers can be adjusted. The gripper is actuated by

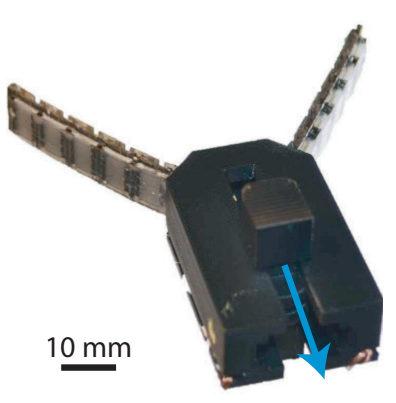

(a)

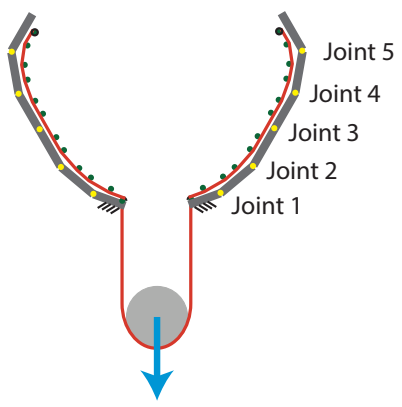

(b)
Fig. 13: Adjustable gripper with adaptive stiffness joints. (a) The Gripper has two fingers each with 5 joints. The stiffness of each joint can be adjusted independently. (b) The schematic of the gripper highlighting the tendon route and the joints position. 
TABLE II: Activating different set of joints results in different grasp modes. For performing the power grasp, all the joints are activated and the fingers conform to the shape of the object. By locking all but the first joint in a desired shape we can preshape the gripper to grasp objects with specific size of shape. For performing precision grasp, the first joint is locked in a desired position based on the size of the object. The second joint is activated and all the other joints are locked at $0{ }^{\circ}$ angle.

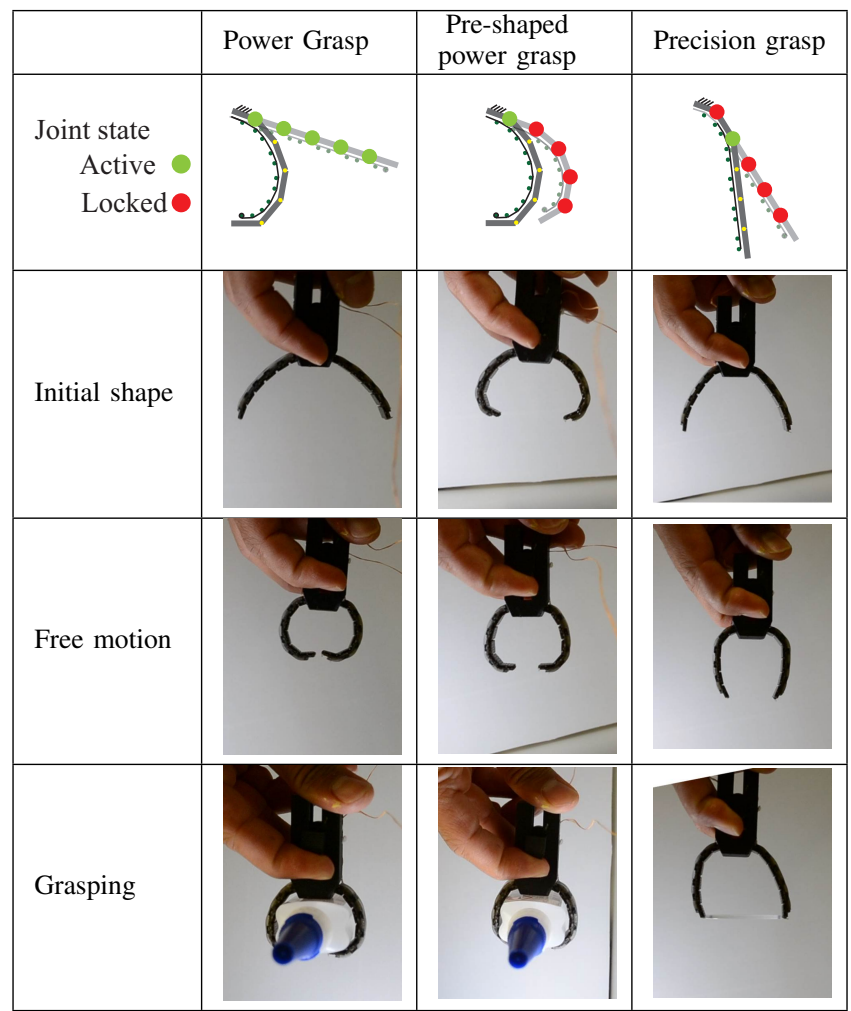

moving a pulley and applying tension to a tendon that runs through both fingers as presented in the schematic of Fig. 13b. In this design, the gripper is actuated manually. Here some preliminary results showing precision and power grasps. At this stage, we only use the joints at locked and completely soft state $\left(\right.$ at $\left.110{ }^{\circ} \mathrm{C}\right)$. A more complex cases with the joint stiffness at intermediate state will be studied in future. Table II presents three different grasp modes. In the first case, all joints are activated and the fingers conform to the shape of the object. The gripper performs power grasp in this mode. In the second mode, the first joint is activated and all the other joints are locked to form a desired shape. In this mode, the gripper can be used for grasping objects of certain shape (or size). The third case presents an example of precision grasp. In this mode the first joint is locked at $45^{\circ}$, to put the finger at the right initial orientation. The second joint is activated and all the other joints are locked at $0^{\circ}$.

The three examples that are presented in this section use only the 2 extremes states for the joint stiffness (completely soft or rigid). Using the model and the characterization results presented in the previous sections and by studying the requirements for a stable grasp, we will study in more detail the stiffness and temperature assignment for the joints in future.

\section{CONCLUSION}

In this paper, the Robogami joint with adjustable stiffness is introduced and its construction process based on the layer by layer manufacturing methodology was presented. The adjustable stiffness joints can be used in under-actuated systems with many DoF for transferring the input energy between different joints according to a desired pattern leading to activation of different synergies.

The compact and scalable Robogami design facilitates further miniaturization and integration of other desired functions in each joint. In the present design the functional layers are adjustable stiffness layer, stretchable heater for controlling the temperature, curvature sensor for monitoring the configuration, and tendons for actuation. The main component in the adjustable stiffness layer is the SMP layer that embeds a stretchable heater. The SMP modulus of elasticity decreases orders of magnitude in temperatures higher than its glass transition. The electrical resistance of the heater is used to estimate and control the temperature of the polymer layer and hence its stiffness for controlling the elastic properties of the joints in the Robogami structure. To improve the strain recovery rate, we embedded the SMP layer inside silicone rubber which resulted in a layer with full shape recovery after elongation of upto $50 \%$. We confirmed the feasibility of the stiffness control based on the temperature estimation from the resistance of the heaters and over 40 times change in the stiffness, 108.0-2.6 N/mm, was demonstrated by changing the temperature from $40{ }^{\circ} \mathrm{C}-110{ }^{\circ} \mathrm{C}$.

To monitor the configuration of the joints and to adjust the parameters in the model and controller we embedded curvature sensors in the structure. These sensors are designed to be compatible with the compact design of the Robogami. To cancel out the temperature effects on the electrical resistance of the sensors, we combined the response of two sensors with similar thermal condition but inverse mechanical loading conditions. The estimation of the angle was shown to be accurate within $2.4^{\circ}$ error bound in 25 loading cycles with different amplitudes.

We used the Robogami joints in an under-actuated finger with three segments and demonstrated configuration control by adjusting the stiffness of the joints through temperature regulation. The kinetostatic model of the finger was proved to capture the behavior of the finger at different temperature settings. The error between the observed joint angle from the curvature sensor and the desired angle can be compensated by further adjusting the tendon displacement and the temperature of the ASLs. We also confirmed the possibility of controlling the overall stiffness of the finger. In the soft mode, the finger conforms to the shape of an object with lower contact force and it can be deformed (back-drivable) with a smaller force. The stiff mode of operation is desirable when larger contact forces are necessary.

Finally we demonstrated the scalability of the Robogami design and manufacturing process by using a scaled down version of the finger in an under-actuated gripper. We demonstrated some preliminary results of different grasp modes. In future, we will use the model for the finger in a more detailed 
study of such a gripper to better exploit the adjustable stiffness of the joints.

\section{ACKNOWLEDGMENT}

This work was supported by Swiss National Center for Competence in Research (NCCR) in Robotics.

\section{REFERENCES}

[1] M. Grebenstein, M. Chalon, W. Friedl, S. Haddadin, T. Wimbck, G. Hirzinger, and R. Siegwart, "The hand of the dlr hand arm system: Designed for interaction," The Int. J. Robot. Res., vol. 31, no. 13, pp. 1531-1555, 2012.

[2] L. Birglen, T. Laliberte, and C. Gosselin, Underactuated Robotic Hands. (Springer Tracts in Advanced Robotics). Springer, 208, vol. 40.

[3] A. M. Dollar and R. D. Howe, "The highly adaptive sdm hand: Design and performance evaluation," The Int. J. Robot. Res., vol. 29, no. 5, pp. 585-597, 2010.

[4] D. M. Aukes, B. Heyneman, J. Ulmen, H. Stuart, M. R. Cutkosky, S. Kim, P. Garcia, and A. Edsinger, "Design and testing of a selectively compliant underactuated hand," The Int. J. Robot. Res., 2014.

[5] M. Ciocarlie, F. M. Hicks, R. Holmberg, J. Hawke, M. Schlicht, J. Gee, S. Stanford, and R. Bahadur, "The velo gripper: A versatile singleactuator design for enveloping, parallel and fingertip grasps," The Int. J. Robot. Res., vol. 33, no. 5, pp. 753-767, 2014.

[6] W. Wang, H. Rodrigue, H.-I. Kim, M.-W. Han, and S.-H. Ahn, "Soft composite hinge actuator and application to compliant robotic gripper," Composites Part B, vol. 98, pp. 397 - 405, 2016.

[7] S. Krut, "A force-isotropic underactuated finger," in Proc. IEEE Int. Conf. Robot. and Autom., April 2005, pp. 2314-2319.

[8] S. Hirose and Y. Umetani, "The development of soft gripper for the versatile robot hand," Mech. Mach. Theory, vol. 13, no. 3, pp. 351 359, 1978.

[9] C. Brown and H. Asada, "Inter-finger coordination and postural synergies in robot hands via mechanical implementation of principal components analysis," in Proc. IEEE Int. Conf. Int. Robot. and Sys., Oct 2007, pp. 2877-2882.

[10] S. A. J. Spanjer, R. Balasubramanian, J. L. Herder, and A. M. Dollar, "Improved grasp robustness through variable transmission ratios in underactuated fingers," in Proc. IEEE/RSJ Int. Conf. Int. Robot. and Sys., Oct 2012, pp. 2289-2294.

[11] S. Wolf and G. Hirzinger, "A new variable stiffness design: Matching requirements of the next robot generation," in Proc. IEEE Int. Conf. Robot. and Autom., May 2008, pp. 1741-1746.

[12] A. Jafari, H. Q. Vu, and F. Iida, "Determinants for stiffness adjustment mechanisms," J. Intelligent \& Robot. Sys., vol. 82, no. 3, pp. 435-454, 2016.

[13] A. Albu-Schaffer, O. Eiberger, M. Grebenstein, S. Haddadin, C. Ott, T. Wimbock, S. Wolf, and G. Hirzinger, "Soft robotics," IEEE Robot. Autom. Mag., vol. 15, no. 3, pp. 20-30, September 2008.

[14] Y.-J. Park, J.-G. Lee, S. Jeon, H. Ahn, J. Koh, J. Ryu, M. Cho, and K.-J. Cho, "Dual-stiffness structures with reconfiguring mechanism: Design and investigation," J. Int. Mater. Sys. Struct., vol. 27, no. 8, pp. 9951010, 2016.

[15] J. Choi, S. Hong, W. Lee, S. Kang, and M. Kim, "A robot joint with variable stiffness using leaf springs," IEEE Tran. Robot., vol. 27, no. 2, pp. 229-238, April 2011.

[16] D. Aukes, B. Heyneman, V. Duchaine, and M. R. Cutkosky, "Varying spring preloads to select grasp strategies in an adaptive hand," in Proc. IEEE/RSJ Int. Conf. Int. Robot. and Sys., Sept 2011, pp. 1373-1379.

[17] B. Vanderborght, A. Albu-Schaeffer, A. Bicchi, E. Burdet, D. Caldwell, R. Carloni, M. Catalano, O. Eiberger, W. Friedl, G. Ganesh, M. Garabini, M. Grebenstein, G. Grioli, S. Haddadin, H. Hoppner, A. Jafari, M. Laffranchi, D. Lefeber, F. Petit, S. Stramigioli, N. Tsagarakis, M. V. Damme, R. V. Ham, L. Visser, and S. Wolf, "Variable impedance actuators: A review," Robot. and Auton. Sys., vol. 61, no. 12, pp. 1601 $-1614,2013$.

[18] A. Firouzeh, S. S. Mirrazavi Salehian, A. Billard, and J. Paik, "An under actuated robotic arm with adjustable stiffness shape memory polymer joints," in Proc. IEEE Int. Conf. Robot. and Autom., May 2015, pp. 2536-2543.

[19] M. A. McEvoy and N. Correll, "Thermoplastic variable stiffness composites with embedded, networked sensing, actuation, and control," $J$. Comp. Mat., 2014.
[20] W. Shan, T. Lu, and C. Majidi, "Soft-matter composites with electrically tunable elastic rigidity," Smart Mater. Struct., vol. 22, no. 8, p. 085005, 2013.

[21] L. Hines, V. Arabagi, and M. Sitti, "Shape memory polymer-based flexure stiffness control in a miniature flapping-wing robot," IEEE Tran. Robot., vol. 28, no. 4, pp. 987-990, 2012.

[22] A. Firouzeh, M. Salerno, and J. Paik, "Soft pneumatic actuator with adjustable stiffness layers for multi-dof actuation," in Proc. IEEE/RSJ Int. Conf. Int. Robot. and Sys., Sept 2015, pp. 1117-1124.

[23] Y. J. Kim, S. Cheng, S. Kim, and K. Iagnemma, "A novel layer jamming mechanism with tunable stiffness capability for minimally invasive surgery," IEEE Tran. Robot., vol. 29, no. 4, pp. 1031-1042, Aug 2013.

[24] T. Ranzani, M. Cianchetti, G. Gerboni, I. D. Falco, and A. Menciassi, "A soft modular manipulator for minimally invasive surgery: Design and characterization of a single module," IEEE Tran. Robot., vol. 32, no. 1, pp. 187-200, Feb 2016.

[25] A. A. Stanley and A. M. Okamura, "Controllable surface haptics via particle jamming and pneumatics," IEEE Trans. Haptics, vol. 8, no. 1, pp. 20-30, Jan 2015.

[26] N. G. Cheng, A. Gopinath, L. Wang, K. Iagnemma, and A. E. Hosoi, "Thermally tunable, self-healing composites for soft robotic applications," Macromol. Mater. Eng., pp. n/a-n/a, 2014.

[27] W. Wang, H. Rodrigue, and S.-H. Ahn, "Deployable soft composite structures," Sci. Rep., vol. 6, p. 20869, 2016.

[28] J. Shintake, B. Schubert, S. Rosset, H. Shea, and D. Floreano, "Variable stiffness actuator for soft robotics using dielectric elastomer and lowmelting-point alloy," in Proc. IEEE/RSJ Int. Conf. Int. Robot. and Sys., Sept 2015, pp. 1097-1102.

[29] I. K. Kuder, A. F. Arrieta, W. E. Raither, and P. Ermanni, "Variable stiffness material and structural concepts for morphing applications," Prog. in Aerospace Sci., vol. 63, pp. 33 - 55, 2013.

[30] H. Tobushi, H. Hara, E. Yamada, and S. Hayashi, "Thermomechanical properties in a thin film of shape memory polymer of polyurethane series," Smart Mater. Struct., vol. 5, no. 4, p. 483, 1996.

[31] A. Firouzeh, Y. Sun, H. Lee, and J. Paik, "Sensor and actuator integrated low-profile robotic origami," in Proc. IEEE/RSJ Int. Conf. Int. Robot. and Sys., Nov 2013, pp. 4937-4944.

[32] L. Birglen and C. Gosselin, "Kinetostatic analysis of underactuated fingers," IEEE Tran. Robot. Autom., vol. 20, no. 2, pp. 211-221, April 2004. 